\title{
Integración supranacional y políticas públicas: la europeización desigual de las políticas sociales
}

\section{Ricard Gomà *}

\section{Introducción}

Este trabajo tiene por objeto considerar los aspectos más relevantes del proceso de europeización de las políticas sociales, entendido como un proceso integral, que replantea no sólo la escala territorial del diseño de políticas, sino también la composición y extensión de la agenda social de actuación pública y los contenidos sustantivos de las políticas de bienestar en Europa. A tal efecto, su estructura presenta dos partes diferenciadas. En la primera, se repasa la dinámica histórica del régimen de bienestar comunitario y se hacen explícitas las bases conceptuales y la hipótesis fundamental del arículo. En la segunda, se analizan las características específicas de la dimensión social de la Unión Europea (UE) respecto a cualquier sistema de bienestar nacionalestatal. Y se desarrolla un esquema explicativo de éstas a partir de una variable institucional: las reglas de toma de decisiones en la UE y de dos factores de contexto: la conexión de la dimensión social al proceso de Unión Económica y Monetaria (UEM) y la compleja heterogeneidad inter-estatal de las políticas sociales.

\section{Las políticas sociales como espacios de regulación del mercado de trabajo y del conflicto distributivo}

Los conceptos de Estado de bienestar y de política social han sido y siguen siendo objeto de discusión y controversia intelectual. Este trabajo no pretende de ninguna manera entrar a fondo en dicha discusión. Sin embargo, cualquier aportación que maneje sistemáticamente ambas nociones debe dejar bien claro sobre qué bases conceptuales se apoya.

En la literatura académica reciente, caracterizaciones amplias y restringidas, tanto de Estado de bienestar como de política social, se cruzan con perspectivas que afirman su equivalencia y otras que sostienen su carácter diferencial. La propuesta conceptual que aqui defendemos es clara: se adoptan nociones amplias y no equivalentes. El Estado de bienestar, de acuerdo con la tesis de OfFE (1984), se considera equivalente al tipo global de estructuración político-económica que emerge en las sociedades avanzadas bajo condiciones de producción y consumo fordistas. Su plasmación vendría dada por un conjunto multifuncional de políticas públicas -económicas y sociales- orientadas a gestionar, de forma simultánea, dos procesos en tensión permanente: crecimiento y distribución. La política social, como componente esencial de dicha estructuración, vendría definida por dos notas básicas. En cuanto a su demarcación sustantiva, el espacio material de la política social se extendería a la regulación de la esfera laboral -condiciones bajo las cuales las personas se insertan o excluyen en los procesos de producción- y a la gestión del conflicto distributivo -tensiones sobre la asignación de valores y recursos entre clases y grupos sociales-. Así pues, la política social vendría a imponer ciertos límites a la mercantilización de la fuerza de trabajo y a crear mecanismos de asignación pública en ciertas parcelas del consumo social y de la obtención de rentas. En cuanto a sus fundamentos normativos, cabría hacer una distinción. Según la perspectiva liberal, el juego libre de las fuerzas de mercado maximizará el bienestar social. Sin embargo, dadas las inelasticidades e imperfecciones informativas que conllevan ciertos bienes sociales (educación, 
sanidad, vivienda, etc.) los mecanismos de mercado tenderán a producir asignaciones sub-optimales. Así pues, la decisión pública colectiva viene justificada -pero también su espacio normativo estrictamente confinado- a alcanzar niveles adecuados de provisión en relación a las preferencias sociales y evitar, de este modo, los impactos negativos de dichas asignaciones sub-optimales sobre los niveles generales de competitividad económica (Kleinmann, Piachaud, 1993, pp. 3-5). Desde una perspectiva crítica, el mercado, dada la subyacente distribución desigual de recursos, se considera un mecanismo de dominación social cuyos resultados expresarán escasamente las preferencias de las clases y grupos desprovistos de poder (LeE, Raban, 1988). En consecuencia, la política social se sustenta normativamente en un doble imperativo: de redistribución (mediante mecanismos de igualación de rentas más allá de las distribuciones mercantiles) y de posibilidad de acceso universalizado a bienes y recursos básicos ${ }^{\text {. }}$.

La opción por esta perspectiva sustantiva y normativa amplia de política social, pero, a su vez, insertada en una concepción global de Estado de bienestar, presenta un mínimo de dos apoyos clave en el terreno de la proyección empírica. Por una parte, supera los criterios definitorios restrictivos, comunes en la literatura anglosajona, que limitan la social policy al sistema de protección social o incluso, en su versión más reductivista, al sistema de garantía de rentas (Bryson, 1992, pp. 33-36). Además, la ampliación conceptual de la política social al binomio empleo-protección posibilita la interrelación empírica y analítica entre política social y económica (Williams, 1989, pp. 20-37. SerRa, 1993). Por otra parte, dicha concepción amplia permite estudiar la conexión entre política social y opciones ideológicas alternativas en el plano izquierda/derecha no exclusivamente en clave de expansión/reducción del gasto social, sino en términos del tipo cualitativo de política social que se formula.

\section{Las fases de evolución histórica de las políticas sociales de la Comunidad Europea}

Las políticas sociales han jugado, de forma permanente, papeles residuales en el proceso de integración comunitaria. La Europa social ha funcionado como discurso de pretensión legitimatoria, pero sus concreciones sustantivas han sido muy débiles y, en cualquier caso, al margen de los elementos centrales de la dinámica de integración. El Tratado de Roma, texto fundacional de la Comunidades, incorporaba una agenda social de extensión muy limitada y no llegaba a romper el monopolio regulativo de los países miembros. Otorgaba a las instituciones europeas simples roles promotores de la cooperación inter-estatal, sobre un abanico reducido de políticas de bienestar. Cabe agregar, a todo ello, que el desarrollo concreto de iniciativas sociales comunitarias se veía sometido a un doble límite institucional: el procedimiento de consulta, que relega al Parlamento Europeo y el sistema de votación por unanimidad, como recurso de poder y baza negociadora clave en manos de los Estados. La situación descrita, aunque dinámica, no sufre alteraciones de alcance hasta finales de los ochenta (GoLD, 1993, pp. 27-35). El proceso desencadenado por el Acta Única (1987) implica una modesta expansión del raquítico régimen de bienestar comunitario en tres niveles: el simbólico (Carta Social Europea de 1989), el normativo (Protocolo Social del Tratado de Maastricht) y el sustantivo (Programas de Acción Social 1989-92 y 1995-97).

Una cierta profundización en el análisis nos conduce a observar, más allá de la constante de debilidad, una dinámica evolutiva configuradora de diferentes etapas históricas en el desarrollo de las políticas sociales europeas. Circunstancias diversas operan como factores explicativos de sus trazos básicos y de los sucesivos puntos de inflexión.

\section{1958-1972: un mercado común sin agenda social}

Entre 1958 y 1972, se da una situación de práctica exclusión de la política social (BREWSTER, TEAGUE, 1989). La homogeneidad de los regímenes nacionales de bienestar de los seis países fundadores, en torno al modelo corporativo, facilita el mantenimiento del conflicto distributivo fuera de la agenda comunitaria. Ello, a su vez, permite no añadir complejidad a un proceso de integración embrionario y refuerza la percepción de la Comunidad en clave económica, como mecanismo orientado a dotar de un marco regulativo supranacional a los sectores clave del modelo fordista de crecimiento.

\section{1973-1980: la irrupción del régimen de bienestar comunitario}

En la cumbre de París (1972), se produce un primer y modesto giro social. Los jefes de Estado y de gobierno deciden proponer a la Comisión el diseño de un Programa de Acción Social a desplegar durante la fase 1973-1980. Las posiciones de los gobiernos socialdemócratas de los setenta en Alemania (Brandt/Schmidt) y el Reino Unido (Wilson/Callaghan) prevalecieron, en la arena política europea, a la todavía débil estrategia neoliberal. Las instituciones comunitarias diseñan políticas en tres ámbitos: el I Programa de Acción contra la pobreza, la regulación de ciertos aspectos del mercado de trabajo (directivas de protección de los trabajadores en caso de regulación de empleo, quiebra y cambio en la propiedad) y la adopción de las tres directivas básicas de igualdad hombre/mujer (salario, 
condiciones laborales y seguridad social). La progresiva consolidación del proceso europeo y la creciente heterogeneidad de los regímenes de bienestar de los Estados miembros (resultado de la ampliación a Dinamarca, el Reino Unido e Irlanda) operaron como factores clave de apoyo.

\section{1980-1988: presiones neoliberales y parálisis social}

A partir de 1980, sin embargo, la nueva hegemonía programática (liderada por los gobiernos Thatcher) y articulada al avance de las posiciones euroescépticas genera un conjunto de fuertes reveses en el desarrollo del frágil régimen de bienestar comunitario (BlanPaIN, ENGEIS, 1993). Sólo en la dimensión de género se producen avances significativos, con el diseño del I Programa sobre Igualdad de Oportunidades y la adopción del principio de acción positiva (1984). Entre 1980 y 1987, el Consejo paraliza todas las propuestas de directiva en el ámbito laboral (BRIDGFord, STTRLING, 1991). Tanto aquellas que pretendían la regulación de derechos individuales en el mercado de trabajo (protección de la contratación no indefinida, reducción de la jornada y permiso de paternidad) como las orientadas a incidir sobre las relaciones industriales colectivas (mecanismos de consulta y representación en las empresas multinacionales). Finalmente, la aprobación a propuesta británica del Programa de Acción sobre crecimiento de la ocupación (1986) implica la asunción, a escala europea, de las propuestas neoliberales de conectar el empleo a la desregulación del mercado de trabajo y al recorte de los gastos en protección social.

\section{1989-1995: génesis de la dimensión social de la UE}

El bienio 1988-89 marca un nuevo y modesto giro social, similar al de principios de los setenta. A los cambios normativos que introduce el Acuerdo de Política Social de Maastricht: potencial de expansión del régimen europeo de bienestar y quiebra del principio decisional de unanimidad, se articula un proceso de desarrollo real de la agenda social comunitaria (Purdiy, Devine, 1994). Las múltiples iniciativas de actuación que conforman dicho proceso pueden analizarse en función del siguiente esquema. Por una parte, se profundiza en áreas de política pública ya consolidadas: directiva-marco de salud laboral, directiva de comités de empresa o reforma de los fondos estructurales. Por otra parte, se produce una extensión de los límites de la política social europea, hacia espacios que van más allá del mundo del trabajo strictu sensu: recomendación sobre convergencia de objetivos en los sistemas de seguridad social (1992), iniciativas sobre exclusión social (III Programa anti-pobreza, programas ERGO y HELIOS) o implicaciones de la igualdad hombre/mujer en la esfera doméstica (Hantrals, 1995).

Los factores explicativos de esta reciente dinámica expansiva son múltiples. Dos perspectivas de análisis han aportado las argumentaciones más elaboradas. De un lado, el enfoque neo- funcionalista sostiene que la culminación del mercado único y el avance hacia la unión monetaria, genera un conjunto de externalidades que, necesariamente, deben colonizar ciertos espacios de política social a escala comunitaria (LeIBFrIED, PIERsoN, 1992). Por otra parte, el enfoque neoinstitucional desplaza la propuesta explicativa hacia la progresiva consolidación de los aparatos burocráticos de la UE. El mercado de políticas sociales comunitarias estaría sujeto, así, a presiones expansivas originadas en el lado de la oferta: preferencias de la socioeurocracia y comportamiento institucional auto-interesado de la Comisión (Cram, 1993, pp. 143-144). Más allá de ambas perspectivas, un análisis de los más recientes conflictos en el seno de la UE nos conduce a otros factores explicativos adicionales: (a) El realineamiento de los partidos cristianos y socialdemócratas en torno al modelo neoliberal, se complementa con la idea de una dimensión social ajustada a la nueva estructura de valores políticos. (b) La ampliación hacia el Mediterráneo acrecienta la heterogeneidad de los regímenes de bienestar y sitúa en el centro de la política social europea la fractura Norte/Sur, ambos aspectos se convierten en motores de ampliación de las oportunidades de regulación social de ámbito comunitario (Amin, TOMAney, 1995).

\section{Los Estados de bienestar y la dimensión social de la UE: diferencias estructurales}

- El desarrollo del Estado de bienestar, en la Europa de la segunda posguerra mundial, supuso una rearticulación de gran alcance en las relaciones entre el Estado, la sociedad y el mercado $^{2}$. Dicho modelo evolucionó a lo largo de más de tres décadas a partir de la interrelación de diferentes variables contextuales: un esquema de producción y consumo fordista; una estructura social homogénea de base clasista con clara expresión política en el sistema de partidos; un amplio consenso normativo reforzado por el imperativo de reconstrucción posbélica, bajo parámetros de solidaridad en clave nacional; y un funcionamiento sostenido de la ecuación keynesiana (BALDWT, 1990). Estas variables estructurales -ninguna de las cuales se halla presente en el marco contextual de la actual dimensión social de la UE- vienen a determinar los trazos básicos comunes adoptados por el modelo social-keynesiano y nacional de bienestar, hasta bien entrada la década de los setenta. Dichos trazos básicos se manifiestan en tres aspectos: (a) Una cierta estructura de la agenda de políticas sociales. Plasmada, a escala estatal, en un régimen pluridimensional de bienestar, conformado por un conjunto interconectado de áreas de actuación sectoriales -empleo, protección social, educación, vivienda-com- 
puestas, a su vez, por un abanico de programas sustentados en valores compartidos (FrancIs, 1993, pp. 43-48). (b) Un triple objetivo estratégico: altas tasas de ocupación masculina estable, la reproducción masiva de la población ocupada y la garantía relativa de rentas de los segmentos excluidos del mercado de trabajo (JaNOSKY, HickS, 1994). (c) Finalmente, dos tipos de politica pública se ajustan a la lógica del modelo y predominan como técnicas de actuación: la provisión directa de servicios y la transferencia de rentas monetarias. La regulación, y más en su dimensión estratégica, queda relegada, en cambio, a un segundo plano (Le Grand, BarTleTt, 1993, pp. 13-34). Ello conectaría directamente con el desarrollo de un modelo administrativo socio-burocrático, monopolista y rígido, en el cual, los soportes organizativos de raíz weberiana se ajustan mal que bien a las nuevas lógicas operativas de orientación taylorista (SuBIRATS, 1991, pp. 15-57).

- Junto con el cambio tecnológico y la segmentación de las pautas sociales de consumo, los procesos de globalización se sitúan en el núcleo del nuevo modelo productivo adoptado por las economías posfordistas (KoTz et al. 1994). La quiebra del marco regulativo protector de los viejos mercados nacionales conlleva impactos masivos en el empleo y en las condiciones de distribución social de la riqueza generada. En Europa Occidental, la progresiva transformación de la Unión Europea en una forma de dominación política de nuevo tipo implica también la posibilidad de realizar (en clave eastoniana) asignaciones vinculantes de valores, a escala comunitaria, sobre un conjunto en expansión de cuestiones económicas y sociales (RICHARDSON, 1994).

En el ámbito económico, la UE ha sellado el compromiso con un modelo de integración basado en valores neoliberales. Las políticas diseñadas para dirigir el proceso incluyen la abolición de todo tipo de barreras a la libre circulación, el alineamiento de las economías nacionales en torno a cuatro criterios de convergencia nominal y la puesta en pie de un nuevo marco único de regulación (TSOuLAKIS, 1993). En el ámbito sociolaboral, el históricamente débil régimen de bienestar comunitario ha cristalizado en la llamada Dimensión Social de la Unión Europea. Un conjunto de políticas y programas sociales configurado a partir de las distribuciones concretas de recursos de poder entre diversos actores, sobre la base de dos ejes semiautónomos de conflicto: la divisoria sustantiva, polarizadora de la regulación pública y las distribuciones de mercado; y la divisoria territorial, polarizadora de la capacidad de decisión entre la Unión, los Estados y las entidades sub-estatales (RHODES, 1991, p. 254; LANGE, 1992, pp. 230-231).

Un análisis preliminar de la dimensión social de la UE ofrece una triple panorámica. En primer lugar, el tipo de reglas decisionales comunitarias en el ámbito social, con una gran debilidad de los componentes democráticos y federales, parece haber generado una estructura de políticas europeas de bienestar incompleta, desigual y predominantemente regulativa. En segundo lugar, el predominio ideológico neoliberal parece haberse trasladado y tomado forma en un ajuste programático global de la dimensión social a los valores políticos imperantes en la UEM. Finalmente, mientras los diversos niveles y tipos de desarrollo nacional no han supuesto un freno para la política de convergencia económico-monetaria, la heterogeneidad en los regímenes de bienestar es crecientemente alegada como un obstáculo insalvable para una hipotética estrategia de convergencia social europea En conjunto, los tres aspectos anteriores pueden ser considerados como la manifestación directa de las tres fuentes estructurales de presión bajo las cuales la dimensión social de la UE está siendo recientemente construida: (a) la dinámica intergubernamental y burocrática que prevalece en la toma de decisiones sociales en la UE, (b) los no cuestionados imperativos de mercado que rigen el proceso de unión económica y (c) las complejas e intensas diferencias entre los sistemas de bienestar de los Estados miembros. Estas realidades difieren radicalmente del marco contextual que arropó el surgimiento y consolidación del modelo clásico de bienestar en Europa. En los próximos apartados, se argumentará que estas diferencias de contexto están en la base de la naturaleza diferencial de la dimensión social europea respecto a cualquier régimen nacional de bienestar.

\section{Las reglas de decisión de la UE y su impacto sobre los límites y el carácter regulativo de la dimensión social}

\section{Unas reglas institucionales de juego en transición}

En el apartado II de este artículo se ha considerado la expansión reciente de la dimensión social de la UE. En el plano legal, se ha sostenido la tesis de que el Protocolo Social de Maastricht permite avanzar hacia un régimen de bienestar pleno a escala comunitaria. En la realidad, observamos que dicha expansión no ha generado más que una estructura muy limitada de políticas sociales. La relación dialéctica entre componentes federales e intergubernamentales, por una parte, y entre componentes democráticos y burocráticos, por la otra, en el marco de toma de decisiones sociales en la UE va a ser tomado como variable explicativa de ello.

El modelo federal-democrático de toma de decisiones sigue teniendo una presencia secundaria en la construcción de la 
Figur! 1

Las politicas de la LEM y de la dimensión social, procesos de adopción de decisiones y reglas de votación

\begin{tabular}{|c|c|c|c|}
\hline \multirow{4}{*}{$\begin{array}{l}\text { Sistemas altemativos } \\
\text { de votación }\end{array}$} & \multicolumn{3}{|c|}{ PROCESOS ALTERNIATTVOS DE ADOPCION DE DECISIONES } \\
\hline & CONSULTA & COOPERACION & CO-DECISION \\
\hline & $\begin{array}{l}\text { - Representación de los trabajadores } \\
\text { - Seguridad social } \\
\text { - Protección al paro } \\
\text { - Política industrial } \\
\text { - Politica fiscal }\end{array}$ & & \\
\hline & - Libertad de establecimiento & $\begin{array}{l}\text { - Derechas de consulta de los trabajadores } \\
\text { - Regulación de las condiciones laborales } \\
\text { - Fomración ocupacional } \\
\text { - Salud laboral } \\
\text { - Igualdad bombre/mujer } \\
\text { - Exclusión social } \\
\text { - Avance hacia la unión monetaria }\end{array}$ & $\begin{array}{l}\text { - Libre movilidad de trabajadores } \\
\text { - Armonización relacionada con el mercado intemo } \\
\text { - Investigación y desarrollo tecnológico } \\
\text { - Redes transeuropeas }\end{array}$ \\
\hline
\end{tabular}

dimensión social de la UE. En la figura 1, el eje horizontal mide la capacidad de incidencia institucional del Parlamento Europeo en el diseño de la dimensión social (JUDGE, EARNSHAw et al., 1994). El eje vertical, mide hasta qué punto la lógica del veto estatal -blindada en el principio de unanimidad- permanece operativa 0 , alternativamente, ha dado paso al principio supranacional de la mayoría cualificada. Cuando las políticas públicas de la dimensión social son ubicadas bajo estas dos coordenadas y son comparadas con las políticas de la UEM, el desajuste queda claramente al descubierto. Estas últimas se rigen por el cruce de la co-decisión con la mayoría cualificada en más casos y en cuestiones más relevantes. Por contra, tres ámbitos clave del régimen de bienestar (seguridad social, protección del desempleo y democracia industrial) siguen anclados en la unanimidad; es más, con la sola excepción de la libre circulación de trabajadores, ninguna otra cuestión social se decide bajo el procedimiento de co-decisión. Ambos aspectos conforman obstáculos institucionales, expresión de voluntades políticas determinadas, hacia la plena consecución de niveles más altos de europeización social.

\section{Una dimensión social incompleta, asimétrica y regula- tiva}

El marco institucional de formulación de políticas sociales comunitarias se halla en la raíz de un régimen de bienestar acotado por tres notas fundamentales: una estructura incompleta (por debajo del mínimo común denominador de los Estados), asimétrica (sesgada hacia la dimensión laboral) y regulativa (sin práctica presencia del complejo de servicios y transferencias).

- La agenda de actuación social de la UE puede estructurarse, a efectos analíticos, en cinco macro-ámbitos; podemos observar como en cada uno de ellos se dan exclusiones temáti- cas (de issues) que ya han transitado hacia la inclusión en las agendas estatales de bienestar: (a) exclusión de políticas de rentas y de regulación de derechos colectivos, en el ámbito laboral; (b) de la política sanitaria más allá de su dimensión ocupacional, en el ámbito de la protección social; (c) de la política de vivienda en el ámbito urbano; (d) de programas de rentas mínimas de inserción en el ámbito de la exclusión social; y (e) de las políticas que inciden sobre el modelo familiar en el ámbito del género. Por otra parte, su naturaleza asimétrica se manifiesta no sólo en la desigual distribución de directivas sociales, con un 78,5 por 100 en el ámbito laboral, sino también en algo más cualitativo: el resto de sectores de actuación se conectan a la esfera del trabajo. La población activa y no el conjunto de la ciudadanía parece conformar el sujeto privilegiado del régimen de bienestar comunitario. De acuerdo al Protocolo Social de Maastricht, el mercado de trabajo parece ser la única fuente de exclusión social: casi todos los programas de acción contra la pobreza selectivizan sus gruposobjetivo en parados de larga duración, mientras escasos programas abordan la discriminación en clave generacional, étnica o de incapacidades físicas o psíquicas (Room, 1991). Por último, el mercado de trabajo se privilegia como mecanismo generador de derechos de protección social: el alto nivel de europeización de las transferencias contributivas contrasta con la nula exportabilidad de las transferencias asistenciales (LEIBFRIED, 1993, pp. 145-148).

- Los modelos nacional-estatales de bienestar han tenido su piedra angular en el complejo de servicios y transferencias configurador del régimen de protección social. Los mecanismos puramente regulativos han sido predominantes, únicamente, en los sistemas de empleo de los modelos corporativos y tradicionales. El régimen de bienestar comunitario, en cam- 


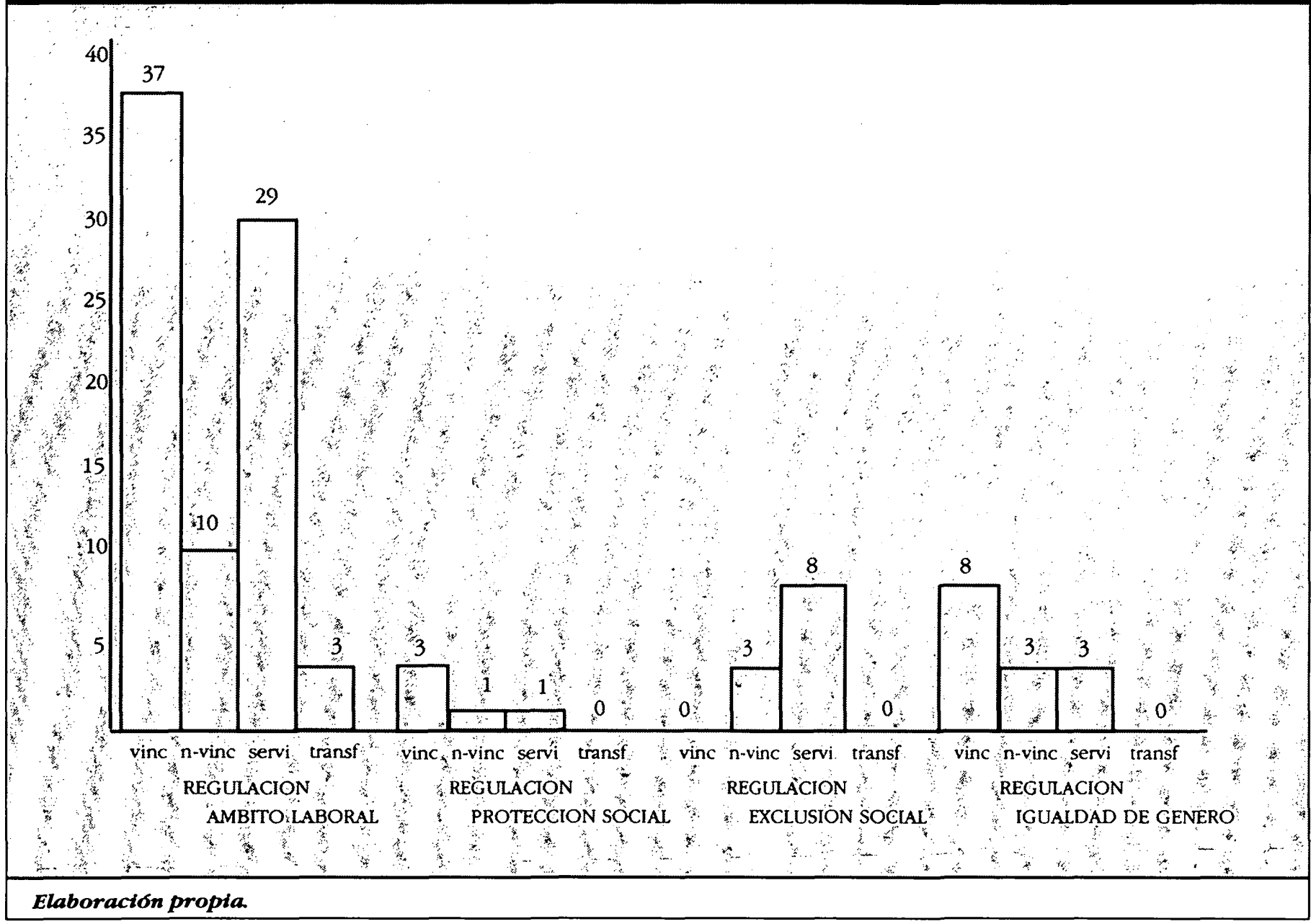

bio, descansa en la regulación (PETERS, 1992). Ello se debe, principalmente, al casi nulo espacio fiscal de la UE: los instrumentos regulativos generan costes económicos soportados por actores no institucionales (sobre todo por sectores industriales). Como muestra la figura 2 , la regulación es el principal componente de acción comunitaria en los ámbitos laboral, de protección social y de género. La provisión de servicios resulta el tipo mayoritario en el ámbito de la exclusión social (Programas Anti-Pobreza), sin embargo, ello no implica relaciones de prestación directas entre la UE y la población. En virtud del enfoque funcional de la subsidiariedad, las instituciones subestatales se erigen en el espacio de gestión apropiado. Los mecanismos de transferencias monetarias quedan limitados a grupos de trabajadores estrictamente selectivizados: los desempleados por procesos de reestructuración diseñados a escala comunitaria en los sectores pesquero, minero y del acero (EC, 1994, pp. 42-45). Por último, no se ha culminado, todavía, ningún convenio colectivo de ámbito UE como resultado del proceso de Diálogo Social iniciado en Val Duchese (1985) y en el marco de la capacidad contractual que Maastricht otorga a los agentes sociales.

\section{La convergencia} económico-monetaria y su impacto sobre los contenidos de la dimensión social

\section{Tres perspectivas sobre el rol de la dimensión social (en el contexto de sus relaciones con la UEM)}

El régimen de bienestar de la UE ha conformado una arena neopluralista de elaboración de políticas sociales. Una densa red de actores interaccionan por medio de dinámicas de conflicto y acuerdo, en el marco de reglas institucionales inestables y de correlaciones de fuerza cambiantes (RICHARD- 
SON, 1994, pp. 21-34). Aun cuando se dan pautas de distribución asimétricas de recursos de poder, ninguna coalición presenta capacidad de imponer el traslado automático de sus intereses al contenido de las políticas. La negociación, en consecuencia, se convierte en mecanismo clave (LANGE, 1992 y 1993, pp. 21-27).

A esta imagen de arena autónoma de formulación de políticas, se superpone una segunda realidad: la dimensión social se encuentra relacionada con el proceso de integración económica bajo criterios de convergencia nominal. Relación que, generalmente, adopta un esquema de dependencia, es decir, las políticas sociales se sitúan bajo la presión de alinear sus contenidos a los parámetros neoliberales y monetaristas de la UEM.

En sintesis, la UEM no excluye la dimensión social comunitaria, pero tampoco se da, entre ellas, un diálogo de igual a igual. Existe un cierto espacio de maniobra en el que se dilucidan las orientaciones de fondo que puede adoptar el sistema de bienestar de la Unión. En este punto y haciendo un cierto ejercicio de simplificación analítica, podemos constatar que la dimensión social se construye sobre tres grandes orientaciones o macro-objetivos, no siempre coincidentes y con plasmaciones desiguales en iniciativas concretas de actuación.

A) El núcleo duro de la coalición neoliberal, conformado por el gobierno británico y la confederación europea de organizaciones patronales, la UNICE, ha venido respaldando la idea de que los beneficios económicos netos de la integración pueden verse erosionados por cierto número de externalidades negativas y barreras no arancelarias. En la medida que algunas de ellas impactan directamente sobre el mercado de trabajo y los procesos distributivos deben ser gestionadas desde iniciativas de política social. Estas, sin embargo, deben quedar normativamente limitadas por criterios eficientistas, sin entrar en el espacio de la redistribución. Desde esta perspectiva se contemplan cuatro tipos de externalidades y barreras de naturaleza social. (a) La espiral de desinversión pública y privada en mecanismos de formación profesional y ocupacional, posibilidad ligada a la opción de contratación de trabajadores cualificados formados fuera del propio país (MARSDEN, 1994). (b) El "dumping" social como factor de distorsión de la competencia. Las empresas que operan con costes laborales bajos obtienen ventajas competitivas en el mercado único. Hecho que impulsa a las empresas que soportan costes socio-salariales altos a una estrategia de presión a su gobierno para la reducción de cargas sociales, bajo amenaza de relocalización territorial. (c) El "regime shopping" como proceso de movilidad de ciudadanos hacia países con transferencias sociales universales. Hecho que provoca, en el Estado receptor, presiones alcistas sobre el gasto social sin generación correlativa de riqueza para financiarlas (LANGE, 1992). (d) La heterogeneidad en los sistemas de seguridad social, factor que funciona como freno a la movilidad, ante la amenaza de pérdida o disminución de derechos como consecuencia de una interrupción en el mecanismo contributivo.

B) Una amplia coalición liderada por la DG V de la Comisión, los gobiernos de los Estados con menos renta y la mayoría cristiana y socialdemócrata del Parlamento Europeo ha venido apoyando una concepción más amplia de la dimensión social de la UE, aun sin poner en entredicbo su ajuste a los imperativos del modelo de convergencia nominal de Maastricht. Desde esta perspectiva, el mercado único es percibido también como generador de ciertas dinámicas dualizadoras entre colectivos sociales y entre territorios de la Unión. En consecuencia, las políticas sociales comunitarias deben corregir las nuevas divisiones sociales y transnacionales del bienestar, por medio del apoyo a los colectivos y territorios afectados por las consecuencias distributivas regresivas del mercado único (CASEY, 1993, pp. 172-78).

C) Finalmente, una constelación de actores configurada por la Confederación Europea de Sindicatos, las fuerzas políticas de la izquierda transformadora en el Parlamento Europeo y un conjunto de ONGs involucradas en la acción colectiva de ámbito europeo en el terreno del bienestar, han propugnado una dimensión social de fuerte componente redistributivo, en diálogo paritario con un modelo reformulado de convergencia económica articulado sobre la cuestión del empleo. Desde esta perspectiva, el régimen europeo de bienestar asistencial propugnado por la Comisión se valora como insuficiente para responder a las desventajas estructurales de los colectivos y territorios periféricos. En consecuencia, se apoya la constitucionalización de los derechos sociales a escala comunitaria y su cristalización por medio de un alineamiento coordinado al alza de los regímenes estatales de empleo y protección social (LeiBfried, PIERSON, 1992).

\section{El contenido de las políticas de la dimensión social}

Se han analizado, en el apartado anterior, las distintas posiciones respecto de la UEM y del tipo de relación de ésta con la dimensión social comunitaria. Ambos factores determinaban las propuestas concretas de los actores sobre el modelo de régimen de bienestar deseado para la Unión. Las preguntas a responder ahora son: ¿hasta qué punto y en qué proporción las tres grandes orientaciones se han trasladado al contenido de las políticas?

La figura 3 muestra la distribución de las más significativas iniciativas de la dimensión social. En relación a las políticas sociales no redistributivas cabe mencionar que la realidad no muestra indicadores palpables de relocalización a consecuencia de estrategias de dumping. Parece evidente que otros factores, como las desventajas comparativas de productividad, contrarrestan las hipotéticas presiones hacia la transferencia norte-sur de capital. Las politicas de la dimensión social ins- 
Figura 3

La dimensión social: orientaciones normativas, actores y contenidos

\begin{tabular}{|c|c|c|}
\hline Modelo de dimensión social & Coalición de apoyo & Politicas más representativas \\
\hline $\begin{array}{l}\text { - Normativamente limitada a criterios eficien } \\
\text { tistas, sin espacio para la redistribución. }\end{array}$ & $\begin{array}{l}\text { - Gobierno conservador británico. } \\
\text { - UNICE (Confederación Patronal Euro- } \\
\text { pea. }\end{array}$ & $\begin{array}{l}\text { - Formación de capital humano. } \\
\text { - Incentivación de la movilidad. } \\
\text { - Reconomiento de cotizaciones y } \\
\text { prestaciones contributivas. }\end{array}$ \\
\hline $\begin{array}{l}\text { - Redistribución-asistencial: ampliada, } \\
\text { pero ajustada a la convergencia nominal. } \\
\text { a }\end{array}$ & $\begin{array}{l}\text { - DG V de la Comisión. } \\
\text { - Gobiernos del sur. } \\
\text { - Mayoría de centro-izquierda en el } \\
\text { Parlamento Europeo. }\end{array}$ & $\begin{array}{l}\text { - Programas para combatir el paro de larga } \\
\text { duración. } \\
\text { - Programaas de acción contra la exclusión } \\
\text { social. } \\
\text { - Programas selectivos de protección al } \\
\text { desempleo. }\end{array}$ \\
\hline $\begin{array}{l}\text { - Redistributiva-universal: ampliada y en } \\
\text { diálogo paritario con una convergencia } \\
\text { reformulada. }\end{array}$ & $\begin{array}{l}\text { - Grupos de izquierda en el Parlamento } \\
\text { Europeo. } \\
\text { - ONGs de ámbito comunitario. }\end{array}$ & $\begin{array}{l}\text { - Directivas de regulación laboral. } \\
\text { - Directivas de igualdad de género. } \\
\text { - Recomendaciones sobre convergencia de } \\
\text { objetivos en protección social y sobre } \\
\text { suficientes recursos en redes de aistencia }\end{array}$ \\
\hline
\end{tabular}

critas en el modelo redistributivo-asistencial se han visto sometidas a tres límites de diferente naturaleza. Un límite fiscal: en 1994 el presupuesto de la UE equivalía al 3 por 100 del gasto público de los Estados miembros y el presupuesto de la dimensión social al 0,9 por 100 del gasto social de los doce (MAJONE, 1993, pp. 160-161). Un límite sustantivo: el selectivismo como principal opción de política social. Los recursos comunitarios no son asignados sobre la base de derechos universales y cobertura extensiva de necesidades, sino dirigidos a grupos-objetivo bien categorizados que enfrenten situaciones de riesgo o necesidad bien acotadas. Finalmente, un límite de estilo: el predominio de un método reactivo de respuesta que conlleva el predominio de políticas sociales paliativas en detrimento de un modelo de bienestar más promocional y preventivo. En el terreno de las políticas redistributivas universales, de forma simultánea a los avances en las dimensiones laboral y de género, caben destacar dos elementos de debilidad claves. Por una parte, el uso de instrumentos de actuación simbólicos, no vinculantes, en ámbitos muy sensibles tanto en clave ideológica como territorial (seguridad social, salarios, rentas mínimas de inserción, educación pre-escolar...) (Cram, 1993, p. 143). Por otra parte, el bloqueo sistemático del Consejo, e incluso de la Comisión, a ciertas propuestas que significarían una paso adelante importante en la concepción amplia del régimen de bienestar comunitario (propuestas de directiva sobre contrataciones no indefinidas y sobre permisos por paternidad, o propuestas de debate sobre el salario mínimo europeo o el sistema europeo de seguridad social).

\section{Diversidad de Estados de bienestar y su impacto sobre los niveles} y la naturaleza de la europeización social

\section{Diversidad y cambio en los sistemas europeos de bienestar}

El modelo clásico de bienestar, más allá de su valor conceptual y analííco, cine su plasmación empírica, estrictamente, al conjunto de rasgos básicos considerados en el apartado III. En el terreno de las realidades, la existencia de un modelo europeo de bienestar no deja de ser un mito que encubre buenas dosis de diversidad interna. Ante ello, en el campo de la ciencia política se desarrollan dos propuestas metodológicas: de un lado, avanzar en el conocimiento de las políticas sociales por medio de estudios nacionales de caso; de otro, diseñar estrategias de análisis comparado. Situados en esta segunda propuesta, la tarea de modelar el análisis comparado de los sistemas europeos de bienestar enfrenta dos dilemas básicos. En primer lugar, optar por un conjunto de indicadores cuya operacionalización muestre capacidad de expresar y medir las variaciones en los contenidos y/o impactos de las políticas sociales (GinsBURG, 1992, pp. 2-13). En segundo lugar, decidir sobre la trans- 
formación de éstos en variables a explicar y, en caso afirmativo, afrontar la necesidad de construir procesos de génesis, a partir de un cierto esquema de relación entre las variables explicativas (BRUGUÉ, GOMÀ, 1996).

\section{A. Pautas de diversidad}

Diferentes autores han optado por diferentes modelos de análisis comparado, con combinaciones específicas de indicadores de contenido e impacto (Castles, Mitchell, 1992). Todos ellos han llegado a dos conclusiones compartidas. En primer lugar, la existencia de fuertes similitudes entre grupos de sistemas nacionales de bienestar, las cuales alcanzan tanto a los contenidos e impactos de las políticas como a los procesos de génesis de las mismas. Dichas similitudes permiten sostener la existencia de tipos transnacionales de bienestar, como variantes empiricas del modelo clásico. En segundo lugar, el acuerdo se ha extendido a la misma estructura tipológica, de carácter tripolar. En clave titmussiana (1974), conformada por los modelos institucional, residual y de rendimientos laborales, y en terminología de ESPING-ANDERSEN (1990) por los mundos de bienestar socialdemócrata, liberal y corporativo-conservador.

Sobre la base de estos dos acuerdos fundamentales, a partir del carácter acumulativo de muchos de los hallazgos parciales de los autores citados, haciendo uso de un marco amplio de variables y, por último, incorporando al universo comparativo los casos de los países europeos del sur que desarrollaron sus sistemas de bienestar en el marco de regímenes autoritarios
(LEIBFried, 1993, pp. 139-143), se hace posible redefinir de forma global los cuatro tipos transnacionales de bienestar y los planos de divergencia principales entre ellos (figura 4).

Una sintesis interpretativa de los cuatro modelos nos conduce a dos ideas fundamentales. En primer lugar, se configura una triple polarización -entre los tipos corporativo, residual y universatcon predominio de los planos de divergencia y coincidencias puntuales. Así, la mayor presencia de subsidios sobre servicios es compartida por los tipos residual (Reino Unido) y corporativo (Estados fundadores de la CEE). Este último limita sus semejanzas con el universal (países escandinavos) a altos índices de gasto y procesos centralizados y confederales de negociación colectiva. Por último, los tipos residual y universal convergen sólo en hacer pivotar el régimen laboral sobre los agentes sociales, con espacios limitados de regulación pública, (CIEMENT, MAHON, 1994). Por otra parte, el tipo tradicional (países mediterráneos), que presenta polarizaciones sistemáticas con el modelo universal, se configura a partir de una cierta articulacion de rasgos propios de los modelos corporativo y residual. En segundo lugar, existe un aspecto adicional, no captado en la figura 4, que define a los diferentes tipos: la lógica concreta de interrelación entre los regimenes laboral y de protección social. En el modelo universal, un mecanismo de protección basado en el principio de ciudadanía social se articula a un sistema de bienestar en el que el Estado se configura como el principal resore de ocupación (LEIBFRIED, 1993, p. 140). En el tipo corporativo, el régimen transferencial contributivo opera como núcleo de un sistema de bienestar globalmente orientado a la protección social, donde, sin embargo, se

\begin{tabular}{|c|c|c|c|c|c|c|c|}
\hline \multirow{3}{*}{$\begin{array}{c}\text { Modelos } \\
\text { de biénestar }\end{array}$} & \multicolumn{5}{|c|}{ Contenidos sustantivos } & \multicolumn{2}{|c|}{ Impactos } \\
\hline & \multicolumn{2}{|c|}{ Variables sectoriales } & \multicolumn{3}{|c|}{ Variables transectoriales. } & \multicolumn{2}{|c|}{ Variable ransectorial } \\
\hline & Régimen laboral & Régimen de transferencias & $\begin{array}{l}\text { Niveles } \\
\text { de gasto, }\end{array}$ & $\begin{array}{l}\text { Niveles } \\
\text { de desmercant. }\end{array}$ & Tipo de actuación & Estructura de rentas & Tipo de impacto: \\
\hline Corporativo & $\begin{array}{l}\text { Tasa sindical media } \\
\text { Negociación central } \\
\text { Densidad regulativa }\end{array}$ & $\begin{array}{l}\text { Contributivo } \\
\text { Conectado a nivel } \\
\text { salarial }\end{array}$ & Altos & Medios & $\begin{array}{l}\text { Predominio de } \\
\text { transferencias }\end{array}$ & $\begin{array}{l}\text { Desigualdad mo- } \\
\text { derada }\end{array}$ & $\begin{array}{l}\text { Reproductor de } \\
\text { status }\end{array}$ \\
\hline Residual & $\begin{array}{l}\text { Tasa sindical baja } \\
\text { Negoc. fragmentada } \\
\text { Escasa regulación }\end{array}$ & $\begin{array}{l}\text { Selectivo. Con com- } \\
\text { probación de me- } \\
\text { dios }\end{array}$ & Bajos & Bajos & $\begin{array}{l}\text { Predominio de } \\
\text { transferencias. }\end{array}$ & $\begin{array}{l}\text { Desigualdad ele- } \\
\text { vada }\end{array}$ & Dualizador. \\
\hline Universal & $\begin{array}{l}\text { Tasa sindical alta } \\
\text { Negociación central } \\
\text { Escasa regulación }\end{array}$ & Universal & Altos & Altos & $\begin{array}{l}\text { Predominio de } \\
\text { servicios }\end{array}$ & $\begin{array}{l}\text { Desigualdad redu- } \\
\text { cida }\end{array}$ & Igualizador. \\
\hline Tradicional & $\begin{array}{l}\text { Tasa sindical baja } \\
\text { Negociación central } \\
\text { Densidad regulativa }\end{array}$ & $\begin{array}{l}\text { Contributivo } \\
\text { Conectado a nivel } \\
\text { salarial }\end{array}$ & Bajos & Bajos & $\begin{array}{l}\text { Equilibrio entre } \\
\text { ambos tipos }\end{array}$ & $\begin{array}{l}\text { Desigualdad ele- } \\
\text { vada }\end{array}$ & $\begin{array}{l}\text { Reproductor de } \\
\text { status }\end{array}$ \\
\hline
\end{tabular}

Fuente: Elaboración propia. 
formulan estrategias de blindaje regulativo de las altas tasas de empleo masculino privado. En el modelo tradicional funciona esa misma orientación global, pero se articula a una esfera laboral con mucha menor capacidad de generación de empleo. Por último, el régimen selectivo del modelo residual se articula a un sistema de bienestar globalmente orientado al trabajo, en el cual las transferencias se vinculan a la búsqueda activa de empleo en un mercado flexible y desregulado (Savage et al., 1994).

\section{B. Pautas de reestructuración}

A lo largo de la última década, un conjunto de procesos socio-económicos emergentes, de alcance internacional, se despliegan sobre los países de la UE. Dichos procesos se hacen tangibles en un abanico de nuevas realidades sobre las que incide directamente la política social. Así, y a modo de ejemplos relevantes, el desempleo afecta a 18 millones de personas en la UE; crecen las tasas de pobreza relativa; el cambio demográfico redobla los índices de dependencia; y la monoparentalidad se sitúa en un 30 por 100 de las familias europeas. Todo ello sitúa a las políticas sociales de los sistemas de bienestar clásicos bajo imperativos de reestructuración. Desde sus viejas lógicas, ninguno de ellos es capaz de dar respuesta adecuada a las realidades sociales emergentes. A partir de esta premisa, puede argumentarse que los sistemas nacionales de bienestar se ven obligados a superar la lógica vigente de policy change incremental, para adoptar una perspectiva de cambio doblemente estratégica ${ }^{3}$. En su alcance, por cuanto afecta a las mismas fronteras de actuación en el terreno social (PIERSon, 1991, pp. 141215). Y en su naturaleza, por cuanto se abren nuevos espacios de conflicto político: son los componentes ideológicos los que van a determinar la orientación de fondo del proceso reestructurador $^{4}$ (MISHRA, 1993, pp. 18-37).

Los regímenes de bienestar de los países de la UE han trazado sus propias rutas de cambio por medio de un conjunto de elecciones sustantivas realizadas en el marco de cinco dimensiones de reestructuración (laboral; de lucha contra la exclusión; de igualdad de género; de relaciones entre empleo, protección y exclusión social; y de relaciones Estado-mercado-sociedad en el diseño de las nuevas políticas). La cuestión clave reside en saber si las pautas clásicas de fractura entre los diversos sistemas de bienestar se han consolidado, han quedado erosionadas o se han disuelto. Así como en saber hasta qué punto se reproduce la distribución de casos nacionales. Los primeros datos parecen mostrar que las rutas de cambio han tendido hacia la complejidad y la multi-direccionalidad. Sin embargo, se apuntan dos regularidades: una de base nacional y otra de lógica sectorial.

Los sistemas de bienestar corporativos y los situados en el eje universalismo-mercado han tendido hacia procesos de cambio internamente coherentes. Un núcleo de valores políticos se ha proyectado a cada uno de los planos de reestructuración de las políticas sociales y se ha reconstruido, de esta forma, una cierta homogeneidad inter-dimensional. Sin embargo, la distribución de casos nacionales no se reproduce de manera mimética. Por otra parte, los sistemas de bienestar tradicionales han mostrado una tendencia a reestructurar sus políticas sociales de forma desarticulada, con orientaciones dispares en función de la dimensión de cambio considerada. El caso español es, en este sentido, paradigmático. En los últimos años se han ido consolidando, de forma simultánea, unas políticas laborales de desregulación masiva, junto a un sistema de protección públicocorporativo y una estrategia de contención de la exclusión social basada en mecanismos tradicionales, entre los cuales, la familia extensa sigue jugando un papel relevante.

Un último apunte nos lleva a subrayar la importancia del elemento sectorial. La esfera laboral y las nuevas pautas de interrelación entre ésta y los regímenes de protección y exclusión social tienden a cambiar de forma previsible y consistente con los valores subyacentes en el régimen clásico. Sin embargo, todo ello se atenúa en los tres planos de cambio restantes. Nuestra hipótesis explicativa, a este respecto, se sitúa en el grado y tipo de relación de cada dimensión con la lógica de clase que fundamenta la política social clásica. Así, el ámbito laboral y sus conexiones con la seguridad social en sentido amplio siguen conformando el núcleo del conflicto distributivo en las sociedades posindustriales. En cambio, los nuevos regímenes de actuación sobre las relaciones de género y de exclusión social, así como el grado de pluralismo introducido en la producción de las políticas sociales, se relacionan de forma escasa con la fractura de clase. Ello explicaría la mayor tendencia al desalineamiento que presentan dichos planos de cambio.

\section{Hacia una integración desigual y estratégica de las politicas sociales}

La compleja y creciente diversidad entre los sistemas de bienestar de los Estados de la UE, juega un papel determinante sobre los niveles y la naturaleza de la europeización de las politicas sociales. Dicho papel, sin embargo, resulta mediado por el conflicto conceptual -vehiculado por actores diversos- en tomo al principio de subsidiariedad. Este principio está sujeto a múltiples interpretaciones, las cuales se estructuran en dos concepciones alternativas. Cuando la subsidiariedad conduce a un patrón de distribución sectorial de responsabilidades entre la Unión y los Estados, se abre la puerta a la exclusión total de determinados ámbitos de actuación social de la agenda comunitaria. En cambio, si el principio en cuestión nos lleva a una asignación intra-sectorial, basada en criterios funcionales de reparto de poderes, el régimen de bienestar de la UE puede extenderse transversalmente, por el conjunto de ámbitos de actuación, concentrándose en funciones de regulación estratégica.

En el doble contexto configurado por las presiones derivadas de la heterogeneidad inter-estatal y por el conflicto epistémico sobre el principio de subsidiariedad, una cuestión aparece fun- 
damental: ¿se está produciendo un desplazamiento de la toma de decisiones sobre el mercado de trabajo y el conflicto distributivo desde el ámbito estatal hacia la arena europea? La respuesta es compleja por un minimo de dos razones. En primer lugar, la idea de europeización, aplicada a una política pública, presenta un carácter complejo, bidimensional. En segundo lugar, nuestros objetos de integración -los sistemas nacionales de bienestarcomprenden diversos ámbitos interconectados de actuación.
La figura 5 expone un modelo simple para el análisis de los procesos de europeización de políticas sociales. El eje vertical (de nivel) permite medir la intensidad competencial de la UE en cada ámbito sectorial. El eje horizontal (de carácter) distingue un tipo de europeización restringida a la esfera de los contenidos básicos de la política, o bien extendida hacia el diseño de las instituciones de implementación en los niveles estatales o sub-estatales. Cruzando ambas dimensiones surgen cuatro

\begin{tabular}{|c|c|c|c|}
\hline \multicolumn{4}{|c|}{$\begin{array}{l}\text { Figura } 5 \\
\text { Un modelo bidimensional de integración de politicas }\end{array}$} \\
\hline & & \multicolumn{2}{|c|}{ Carácer de la integración } \\
\hline & & Contenidos hásicos & Diseño institucional \\
\hline \multirow{4}{*}{ Nivel de la integración } & \multirow{4}{*}{$\begin{array}{l}\text { Europeización alta } \\
\text { Alineación } \\
\text { Coordinación } \\
\text { Europeización baja } \\
\text { Intercambio } \\
\text { Aislamiento }\end{array}$} & Convergencia de objetivos & Armonización de políticas \\
\hline & & $\begin{array}{l}\text { Igualdad de sexos } \\
\text { Seguridad social }\end{array}$ & $\begin{array}{l}\text { Salud laboral } \\
\text { Libre circ. trabajadores }\end{array}$ \\
\hline & & Intercambio de ideas & Transferencia de políticas \\
\hline & & $\begin{array}{l}\text { Exclusión social } \\
\text { Sanidad }\end{array}$ & $\begin{array}{l}\text { Formación ocupacional } \\
\text { Vivienda }\end{array}$ \\
\hline
\end{tabular}

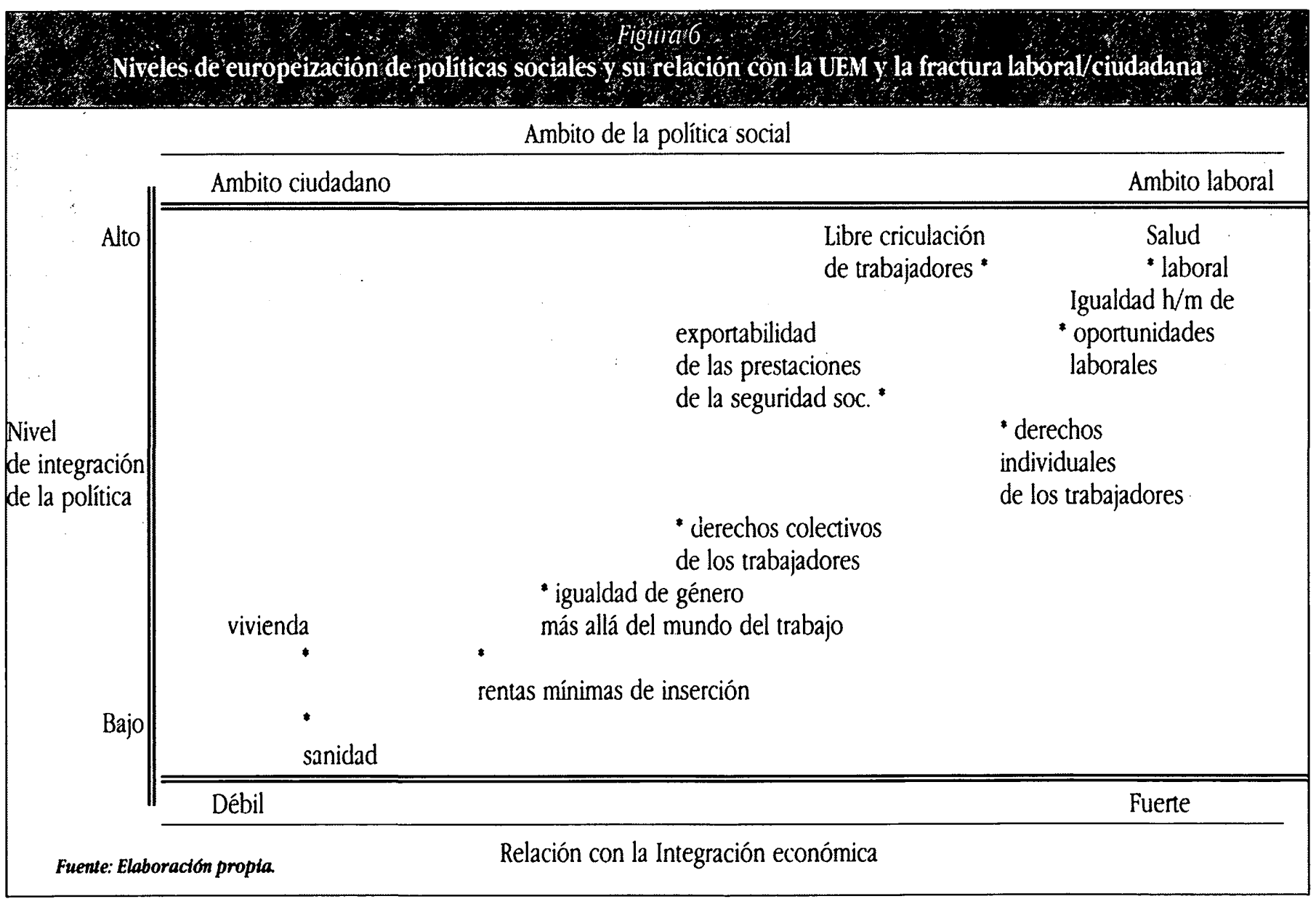


espacios conceptuales, como expresión global del nivel y el carácter de la europeización de cada área de política social.

El régimen de bienestar de la UE, en su actual configuración, no permite ser unitariamente ubicado. El Acuerdo de Política Social de Maastricht ofrece base normativa suficiente para intensificar los procesos de integración. Sin embargo, la realidad difiere en gran medida de esa posibilidad. La europeización social, aunque desigual, presenta dos regularidades básicas. Y es aquí cuando la compleja y creciente diversidad entre los sistemas de bienestar de los Estados de la UE entra en juego como factor explicativo. (a) Se oberva una progresión desde el tipo armonizador bacia el estratégico de integración, paralelo al relevo del enfoque sectorial por el funcional de subsidiariedad, durante los últimos años. En efecto, se ha generalizado la gestión descentralizada de políticas sociales, tendente a incrementar la sensibilidad de los programas a realidades sociales específicas, altamente cambiantes y fragmentadas. Ello cierra la puerta a cualquier pretensión armonizadora central, reintroductora de rigideces institucionales. Sin embargo, la escala transnacional de las cuestiones sociales emergentes (nuevos procesos de exclusión, paro estructural, alteración de los términos de relación intergeneracional...) ofrece la posibilidad de explorar trayectorias y modelos alternativos de integración: básicamente, aquellos articulados en torno a la centralización de las funciones de regulación social estratégica y a la convergencia flexible de objetivos en el ámbito de la UE.

(b) Se constatan fuertes obstáculos trans-sectoriales hacia niveles altos de europeización. Sin embargo, la figura 6 muestra la polarización de ámbitos selectivos del régimen de bienestar comunitario en niveles altos de integración. Y, aún más significativo, se observa un doble patrón de comrelación entre el grado de europeización, la funcionalidad respecto del proceso de convergencia económica y la conexión de la política pública a la esfera laboral. Así, se puede observar el contraste entre la integración alta de las transferencias contributivas de la seguridad social y la integración residual de los sistemas asistenciales de garantía de rentas; patrón de contraste que se reproduce entre salud laboral y sanidad en general, o entre igualdad de género en la esfera del trabajo y en la esfera familiar. Finalmente, en la esfera laboral, la europeización es.más elevada cuando se trata de regulaciones de derechos individuales de los trabajadores y mucho más residual cuando se entra en las relaciones industriales colectivas.

\section{Conclusiones}

La dimensión social de la Unión Europea representa un nuevo modelo de estructuración de las políticas de bienestar en Europa. Entre 1958 y 1992, el desarrollo de las políticas sociales comunitarias constituyó una débil realidad factual bajo severos limites normativos. El Acuerdo de Política Social de Maastricht abrió la puerta legal hacia la plena europeización social sobre una base expansiva de políticas públicas. Sin embargo, el análisis empírico de los aspectos configuradores básicos de la dimensión social de la UE hacia mitad de los noventa nos aporta un mínimo de tres conclusiones. (1) El régimen de bienestar comunitario muestra una composición interna incompleta, con exclusiones temáticas inéditas a escala estatal, asimétrica, con un fuerte sesgo hacia la consideración de la población activa y no del conjunto de la ciudadanía como verdadero sujeto de las políticas sociales y regulativa, con una débil presencia de actividades prestacionales, transferenciales o de negociación colectiva. (2) La dimensión social se sitúa bajo la presión de alinear sus contenidos a los parámetros neoliberales y monetaristas de la UEM. Dicha presión es vehiculada por diferentes coaliciones de actores que operan como instrumentos de transferencia de sus propias concepciones al contenido de las políticas sociales comunitarias. Como resultado, observamos una dimensión social de contenido redistributivo bajo, con más énfasis en programas asistenciales y selectivos que en derechos universales. (3) Respecto a la fractura territorial, hemos observado una europeización desigual, que correlaciona, en sus niveles máximos, con aquellas políticas sociales funcionales a la UEM y conectadas al mundo del trabajo. Por otra parte, la integración ha consolidado su naturaleza estratégica, derivada de la subsidiariedad funcional, a expensas de las estrategias armonizadoras de etapas anteriores. Este conjunto de rasgos configuradores básicos, que refuerzan la idea de especificidad del régimen de bienestar de la UE, han sido explicados a partir de una variable institucional y de dos factores estructurales de contexto. (a) El predominio de una escasamente erosionada lógica intergubernamental en el proceso de toma de decisiones sociales en la UE. Ello comporta unos altos índices de poder estatal frente al Parlamento y la Comisión y, por lo tanto, límites al avance de la transferencia de más espacio sustantivo y fiscal del ámbito nacional hacia el régimen de bienestar comunitario. (b) La relación de la dimensión social, insertada en un esquema de dependencia parcial, con el proceso de integración económica bajo criterios de convergencia nominal. La presión hacia la redistribución que la reconstrucción nacional solidaria ejercía sobre el Estado de bienestar posbélico, se convierte, hoy por hoy, en presión hacia el eficientismo mercantil en el marco de la dimensión social de la UE. (c) La compleja y creciente diversidad de unos sistemas nacionales de bienestar que se transforman en múltiples direcciones y que, dada la condición hegemónica de los Estados como actores en el proceso de integración, limitan de forma constante el potencial unificador que pudiera tener una dimensión social europea plenamente desarrollada. 


\section{- Universitat Autònoma de Barcelona}

1 En el campo del análisis económico de ta polrica social, dos escuetas de pensamiento se relacionan estrechamente con cada una de las perspecivas normativas consideradas. Por un tado, ta microeconomía neoctásica (ta fuente teórica del monetarismo y de las politicas ortodoxas de ofena) "stants from tbe premise tbat unnestrained markets tend to maximize uelfare (GNSBURG, 1992, p. 12). Por oura parte, la macro-economia keynesiana considera la fiscalidad y el gasto público como instrumentos estabilizadores contra la dinámica cíclica de inflación y paro. En este marco, el gasto social "may be used to generate demand and bence employment and economic growtb" (GiNsBuRG, 1992, p. 12). Sin embargo, es erróneo asumir que existe una relación intrínseca entre keynesianismo y expansión de las políticas de bienestar. La coincidencia histórica de la teoria keynesiana con la hegemonía socialdemócrata en la Europa Occidental de post-guerra ayuda a comprender el equivoco. Sin embargo, un keynesianismo de nueva derecha emergió, durante los ochenta, en los EEUU. La política económica de las administraciones republicanas confió en la expansión del gasto militar como mecanismo contracíclico.

${ }^{2}$ No se deben menospreciar los roles socio-conómicos del Estado europeo occidental en períodos históricos previos. Dichos roles fueron de vital importancia, durante la fase del capitalismo comercial, en el apoyo a la construcción de mercados nacionales unificados. En el Estado alemán bismarckiano, las políticas sociales constituyeron la fuerza motriz de la unificación política. Finalmente, en las décadas de entreguerras, el Salsjobaden sueco allanó el camino al corporatismo social como modo de regulación societal extemo al mercado, y el gobiemo (liberal) británico estableció un sistema fiscal progresivo. Sin embargo, no es hasta después de la П Guerra Mundial, cuando las políticas socio-económicas de intervención masiva se convierten en factor estructural del capitalismo avanzado.
${ }^{3}$ En la literatura académica reciente, pueden distinguirse dos grandes lineas teóricas que ensayan sendas explicaciones sobre los recientes cambios en los sistemas de bienestar. Los autores encuadrados en la primera de ellas tienden a poner el acento en la "lógica de la contracción". Su tesis central consiste en afirmar que el desmantelamiento de las politicas sociales a manos de las fuerzas del mercado ha constituido el rasgo predominante a lo largo de la última década (por ejemplo, Pierson, 1991, 1994). Desde posiciones teóricas no coincidentes, otros autores han tendido a enfatizar la "lógica de la reestructura ción". En este caso, los factores socio-económicos habrían determinado el colapso del bienestar keynesiano, pero en ningún caso la dirección del cambio posterior. El resultado de éste resultaría más complejo: coaliciones específicas de actores pueden haber reestructurado las políticas sociales, en diferentes contextos estatales o regionales, de acuerdo a valores político-ideológicos alternativos (por ej., Jornson, 1987, Brugué, 1993).

${ }^{4}$ Desde luego, la operatividad de los componentes ideológicos depende de la correlación de fuerzas que se establezca en cada coyuntura, y por tanto de la capacidad real de incidencia de las diferentes coaliciones en el proceso de producción de las políticas sociales. De entre los recursos que dotan de posiciones de poder a los diferentes actores políticos, la literatura acadé mica ha prestado un gran interés, recientemente, al rol de los recursos cog. nitivos. En análisis de políticas públicas, el concepto de "comunidades epistémicas" (redes de actores con gran capacidad de producción de conocimiento y movilización de recursos cognitivos) está siendo crecientemente utilizado como factor explicativo de los contenidos sustantivos de las políticas, así como del policy change. RaDaELLI (1995 p.159-179) realiza una excelente síntesis.

\section{Bibliografia}

Amin, A., TOManeY J. (1995) Behind the myth of European Union. Routledge, Londres.

BALDWIN, P. (1990) The politics of social solidarity. Cambridge University Press, Cambridge.

Blanpain, R., Encels, Ch. (1993) European Labour Law. Deventer: Kluwer.

Brewster, CH., Teague, P. (1989) EC social policy. Its impact on the United Kingdom. IPM.

BRIDGford, J., STtRung, J. (1991) "Britain in a Social Europe: industrial relations and 1992", en Industrial Relations Joumal, vol. 22, n. ${ }^{\circ} 4$.

BRUGuÉ Q, Gomi R. (1996) "El impacto de los partidos sobre las políticas sociales. Una perspectiva comparada". De próxima publicación en Revista de Estudios Políticos.

BRYSON, L. (1992) Welfare and the State. London: Macmillan.

CASTLFS, F., MrTCHEL, D. (1992) "Identifying welfare state regimes: the links between politics, instruments and outcomes", en Governance, vol. $5, n .{ }^{2} 1$.

CASFY, B. (1993) "Employment promotion", en Gold, M. The Social Dimension. London: Macmillan.

Clement, W., MaHON, R. (1994) Swedish social democracy. A model in transition. Toronto: Canadian Scholars' Press.

Cram, L. (1993) "Calling the tune without paying the piper?", en Policy and Politics, vol. 21, 2.

EC (1994) Community Social Policy.

ESPING ANDERSEN, G. (1990) The three worlds of welfare capitalism. Cambridge: Polity Press.
Francis, J. (1993) The politics of regulation. Oxford: Blackwell.

GINSBURG, N. (1992) Divisions of welfare. London: Sage.

Gow, M. (1993) The Social Dimension. London: Macmillan.

Hantrais, L. (1995) Social Policy in the European Union. London: Macmillan. JANOSKY, T., HICKS, A. (1994) The comparative political economy of the welfare state. Cambridge: Cambridge University Press.

JudGE, D., EARNSHaw, D., COWAN, N. (1994) "Ripples or Waves: the European Parlament in the EC policy process", en Joumal of European Public Policy, vol. 1, n. ${ }^{\circ} 1$.

Kleinman, M., Piachaud, D. (1993) "European social policy: conceptions and choices", en Jourmal of European Social Policy, vol. 3, n. ${ }^{\circ} 1$.

Kotz et al. (1994) Social Structures of Accummulation. Cambridge: Cambridge University Press.

LAivGE, P. (1992) "The politics of the social dimension", en Sbragia, A. EuroPolitics. Washington: Brookings Institution.

LaNGF, P. (1993) "Maastricht and the social protocol: why did they do it?", en Politics and Society, vol. 21. n. ${ }^{9} 1$.

Lf.E P., RABAN C. (1988) Welfare Theory and Social Policy. London: Sage.

LẸBFRIED, S. (1992) "Europe's could-be social state: social policy in European integration after 1992, en Adams, W. Singular Europe. The University of Michigan Press.

LeibfrifD, S. (1993) "Towards a European welfare state ?", en Jones, C. New perspectives on the welfare state in Europe. London: Routledge.

Leibfried, S., Pierson, P. (1992) "Prospects for social Europe", en Politics and Society, vol. 20. 


\section{Bibliografia}

LE GRAND, J., BARTLETT, W. (1993) Quasi-markets and social policy. London: Macmillan.

Majone, G. (1993) "The European Community between social policy and social regulation", en Journal of Common Market Studies, vol. 31 n. ${ }^{9} 2$.

MaRSDEn, D. (1994) "The integration of European Labour Markets", en EC, "European Integration and the European Labour Market". (Social Europe, supplement 1).

MISHRA, R. (1989) The welfare state in capitalist society. London: Harvester.

Mishra, R. (1993) "Social policy in the postmodern world", en Jones, C. (ed) New perspectives on the welfare state in Europe. London: Routledge.

OFFE, C. (1984) Contradictions of the welfare state. London: Hutchinson.

PETERS, G. (1992) "Bureaucratic politics and the institutions of the EC", en Sbragia A. Euro-Politics. Washington: Brookings Institution.

PIERSON, CH. (1991) Beyond the welfare state? Cambridge: Polity Press.

PIERSON, P. (1994) Destmantling the welfare state? Cambridge: Cambridge University Press.

Purdly, D., Devine, P. (1994) "Social Policy", en Artis, MJ., Lee, N. The economics of the European Union. Oxford: Oxford University Press.
RaDAELL, C. (1995) "The role of knowledge in the policy process", en Jourmal of European Public Policy, vol. 2, n.․ 2.

RICHARDSON, J. (1994) "EU water policy: uncertain agendas, shifting networks and complex coalitions", en Environmental Politics, vol. 4, n." 4.

RHODEs, M. (1991) "The social dimension of the single european market: national versus transnational regulation", en European Jourmal of Political Research, 19.

Room, G. (1991) Towards a European Welfare State? Bristol: School for Adv. Urban Studies.

Savage, S., Atkinson, R., Robins, L. (1994) Public Policy in Britain. London: Macmillan.

Serra, A. (1993) "Redefinint la producció de benestar" (Ponencia no publicada)

Trrmuss, R. (1974) Social Policy. London: Allen and Unwin.

TsoulakIS, L. (1993) The new European economy. Oxford: Oxford University Press.

WIшамs, F. (1989) Social Policy: a critical introduction. Cambridge: Polity. 